\title{
Design and Implementation of Neuro Fuzzy model for Software Development Time Estimation
}

\author{
Shina Dhingra \\ Student \\ DAV Institute of Engineering \& \\ Technology \\ Kabir Nagar, Jalandhar \\ PUNJAB
}

\author{
Palvinder Singh Mann \\ Assistant Professor \\ DAV Institute of Engineering \& \\ Technology \\ Kabir Nagar, Jalandhar \\ PUNJAB
}

\begin{abstract}
To develop a project successfully, it is important for any organization that the project should be completed within budget, on time and the project should have requisite quality. This paper presents an Adaptive Neuro-Fuzzy Approach for Software Development Time Estimation. This proposed technique is aimed at building and evaluating a Neuro - fuzzy model using three (3) membership functions (MFs) for software project development time. The forty one modules were used as a data set. Our proposed approach for Neuro fuzzy using 3 membership functions i.e. Gaussian MF (GMF), Triangular MF (Tri MF) and Trapezoidal MF (Trap MF) is compared with neural network models and the results show that values of various relative error parameters for Neurofuzzy is lower than the values of parameters applying neural network.
\end{abstract}

\section{General Terms}

Neuro Fuzzy, Software Time Estimation.

\section{Keywords}

Adaptive Neuro Fuzzy Inference System (ANFIS), Neural Network, Fuzzy Logic, Prediction, MRE, MMRE, BRE, Development Time (DT), Membership Function (MF).

\section{INTRODUCTION}

Software industries are one of the largely growing industries in recent years. Success of these growing industries plays a key role to the world balanced economy. For the success of the software industries, management of the industries has to plan a software development project. The neural network research started in the 1940s, and the fuzzy logic research started in the 1960s, but the Neuro-fuzzy research area is relatively new [32]. The objective of this paper is to present a possible way of combining fuzzy logic and neural networks for achieving higher accuracy. Once the concept of fuzzy logic is incorporated into neural network, the result is a Neuro-fuzzy system that combines the advantages of both techniques.

A software tool (MATLAB 7.10) was used to process the Neuro-fuzzy systems. Accurate time estimation is a crucial skill in project management. Without it, you won't know how long your project will take, and you won't be able to get commitment from the people who need to sign it off. Even more importantly for your career, sponsors often judge whether a project has succeeded or failed depending on whether it has been delivered on time and on budget. To have a chance of being successful as a project manager, you need to be able to negotiate sensible budgets and achievable deadlines. Once you've estimated the time needed for each task, you can prepare your project schedule. Add your estimates to the draft activity list that you produced in the second step, above. You can then create a Gantt chart to schedule activities and assign resources to your project; and to finalize milestones and deadlines. There are different approaches that you can use to estimate time. Bottom-Up estimating, Top-Down estimating, comparative estimating, parametric estimating and three Point estimating. There are various steps to estimate time accurately. Integration of neural networks, fuzzy logic and algorithmic models into one scheme has resulted in providing robustness to imprecise and uncertain inputs. In this paper, we present an adaptive Neuro fuzzy inference system (ANFIS) for software development time estimation. In the proposed method accurate estimation of software development time will be done and the results of Neuro Fuzzy approach will be compared with different types of neural network models based upon various parameters such as Magnitude of Relative Error (MRE), Mean Magnitude of Relative Error (MMRE), Balanced Relative Error (BRE) and Prediction (Pred).

\section{RELATED WORK}

Lopez Martin et al. [14] proposed a fuzzy logic model for development time estimation. Ting su et al. [33] described an enhanced fuzzy logic model for the estimation of software development effort which had the similar capabilities as the previous fuzzy logic model in addition to enhancements in empirical accuracy in terms of MMRE. Abbas Heiat [34] used artificial neural network techniques like RBF (Radial Basis Function) and MLP (Multi Layer Perceptron) for software development effort. The main goal of this paper is to evaluate software development time using an adaptive Neuro fuzzy approach.

\section{METHODOLOGY}

The main goal of this paper is to evaluate software development time using an adaptive Neuro fuzzy approach. In this paper an Adaptive Neuro Fuzzy Inference System (ANFIS) tool is used. The network is trained by using learning algorithm i.e. Hybrid Approach (combination of back propagation and least mean square algorithm). This methodology consists of four steps: 1) Loading of Training Data and 2) Generating Fuzzy Inference System 3) Training of ANFIS 4) Development Time Estimation. 


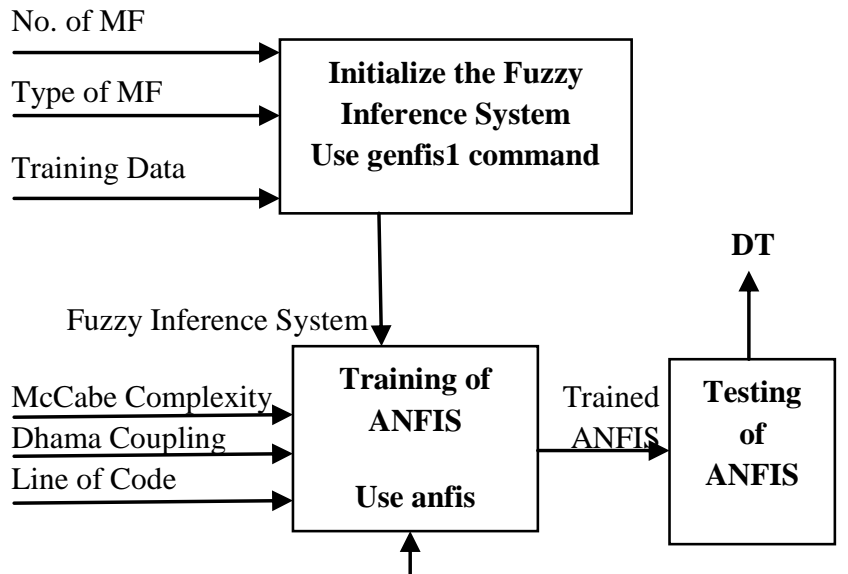

Other Parameter for learning(i.e.no of epochs, learning algorithm etc.)

Fig 1: Proposed Model

\section{Pseudo - Code of Methodology}

Begin:

Step I; Determine the inputs of the model;

Collect a data set; Divide the data into two sets: Train data set, and the other one for evaluating the validity of the estimated model, called the test data set.

Step II; Generate ANFIS model;

[Define no of Membership functions] numMFs; [define type of Membership functions] mfType; [define no epoch] epoch_n;

[Generate Fuzzy Inference System structure from data using grid partition] in_fis=genfis1 (trnData, numMFs, mfType); [Training routine for Sugeno-type Fuzzy Inference System (uses a hybrid learning algorithm)]

out_fis= anfis (trnData,in_fis,epoch_n);

Step III; Evaluate the value of Development Time;

For each individual test data

For $\mathrm{i}=1$ to total test data

[Evaluate the value of Development Time ]

$\mathrm{dt}(\mathrm{i})=$ evalfis(inpData,fis);

Next i ;

Step IV; Evaluate the Value of MRE from result obtained by step III;

For MRE of each individual test data

For $\mathrm{i}=1$ :to total test data $\operatorname{mre}(1, \mathrm{i})=\mathrm{abs}(($ Actual dt(i) $-\mathrm{dt}(\mathrm{i})) /$ Actual $\operatorname{dt}(\mathrm{i}))$;

Next i ;

Step V; Evaluate the Value of MMRE and PRED from result obtained by step IV;
For MMRE and PRED of each individual test data

Initialize mmre $=0$, pred $=0$;

For $\mathrm{i}=1$ :to total test data mmre $=$ mmre + mre $(i)$;

IF $($ mre $(\mathrm{i})<=.25)$

pred $=$ pred +1 ;

EndIF

Next i ;

MMRE $=($ mmre/(total test data $)) * 100$;

PRED $=$ pred/(total test data);

Step VI; Evaluate the Value of BRE from result obtained by step III;

For BRE of each individual test data

For $\mathrm{i}=1$ :to total test data

bre $(1, \mathrm{i})=\mathrm{abs}(($ Actual $\mathrm{dt}(\mathrm{i})-\mathrm{dt}(\mathrm{i})) / \min ($ Actual $\operatorname{dt}(\mathrm{i}), \operatorname{dt}(\mathrm{i})))$;

Next i ;

END

\subsection{ANFIS Method}

The proposed method estimates the software development time accurately by proposed Adaptive Neuro Fuzzy Inference System (ANFIS) as it is a combination of Fuzzy Logic and Neural Network so ANFIS takes advantages from fuzzy logic and neural network. This ANFIS constructs a Fuzzy inference system by using given training data set whose membership function parameters are adjusted by back propagation algorithm or in combination with least square type of method. Fig.2 shows a high level diagram of the proposed ANFIS. Inputs and their membership functions appear to the left of the ANFIS structural characteristics, while outputs and their membership functions appear on the right.

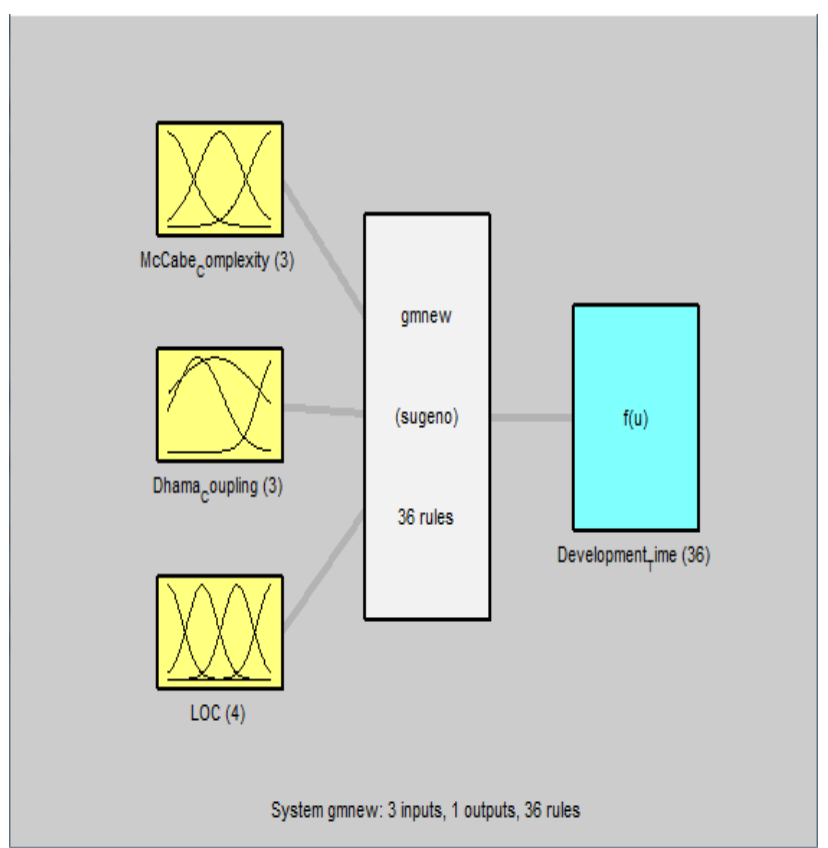

Fig 2: Diagram of Proposed ANFIS for Gaussian MF 


\subsection{Performance Evaluation Metrics}

The following evaluation metrics are adapted to assess and evaluate the performance of the time estimation models.

$\square$ Magnitude of Relative Error (MRE)

$$
M R E=\frac{\mid \text { Actual Time-Estimated Time } \mid}{\text { Actual Time }} \times 100
$$

\section{Mean Magnitude of Relative Error (MMRE)}

$M M R E=\frac{1}{n} \sum_{\mathrm{i}=1}^{\mathrm{i}=\mathrm{n}} \frac{\mid \text { Actual Time-Predicted Time } \mid}{\text { Actual Time }}$

The MMRE calculates the mean for the sum of the MRE of $n$ projects. Specifically, it is used to evaluate the prediction performance of an estimation model.

\section{Prediction Level (PRED)}

$\operatorname{PRED}(l)=\frac{k}{n} \times 100$

where 1 is the maximum MRE of a selected range, $n$ is the total number of projects, and $\mathrm{k}$ is number of projects in a set of $n$ projects whose MRE $<=1$. PRED calculates the ratio of projects' MREs that falls into the selected range (1) out of the total projects. (e.g. $\mathrm{n}=100, \mathrm{k}=80$, where $\mathrm{L}=\mathrm{MRE}<=30 \%$ : $\operatorname{PRED}(30 \%)=80 / 100=80 \%)$.

\section{Balanced Relative Error (BRE)}

$$
\operatorname{BRE}(\%)=\frac{\mid \text { Estimated Time }- \text { Actual Time } \mid}{\min \left(T, T^{\prime}\right)} \times 100
$$

Where $\mathrm{T}=$ estimated time and $\mathrm{T}^{\prime}=$ actual time

\subsection{Training}

The objective of Hybrid training algorithm is to minimize the approximation error. In order to overcome certain problems hybrid approach is used i.e. combination of back propagation algorithm and least mean square method. The 25 projects are used for training that is chosen randomly and 11 projects are used for testing. When fuzzy inference file is trained no. of epochs are used which are used to minimize the error. More the no. of epochs lesser will be the error. In ANFIS epochs are used to adjust the weights in order to minimize the error. To create a fuzzy inference system file genfis 1 command is used and for training of that file anfis command is used which takes certain inputs.

\section{RESULTS AND DISCUSSION}

Testing was carried out on a system with Intel (B core (i3), $2.93 \mathrm{GHz}$ with $2 \mathrm{~GB}$ RAM and implemented using MATLAB (7.10). We have divided the entire dataset into two sets, training set and testing set in the ratio of $100 \%$ and $20 \%$. Training set consists of data from 25 projects selected randomly and testing set consists of 11 project samples that were used later on for testing.

Table 1 summarizes the development time estimates as obtained for Neuro fuzzy model using different membership functions

Table 1.The DT obtained by different MF using ANFIS model

\begin{tabular}{|l|l|l|l|l|}
\hline $\begin{array}{l}\text { Project } \\
\text { No. }\end{array}$ & $\begin{array}{l}\text { Actual } \\
\text { DT }\end{array}$ & $\begin{array}{l}\text { Estimated } \\
\text { DT using } \\
\text { Gaussian } \\
\text { MF for } \\
\text { Neuro } \\
\text { fuzzy }\end{array}$ & $\begin{array}{l}\text { Estimated } \\
\text { DT using } \\
\text { Triangular } \\
\text { MF using } \\
\text { Neuro } \\
\text { fuzzy }\end{array}$ & $\begin{array}{l}\text { Estimated } \\
\text { DT using } \\
\text { Trapezoidal } \\
\text { MF using } \\
\text { Neuro } \\
\text { fuzzy }\end{array}$ \\
\hline 31 & 19 & 19.0021 & 19.0027 & 19.0000 \\
\hline 32 & 13 & 13.0004 & 13.0001 & 13.0000 \\
\hline 33 & 12 & 11.9984 & 11.9997 & 12.0000 \\
\hline 34 & 12 & 11.9977 & 11.9974 & 12.0000 \\
\hline 35 & 21 & 21.0000 & 21.0000 & 21.0000 \\
\hline 36 & 21 & 21.0000 & 21.0000 & 21.0000 \\
\hline 37 & 19 & 19.0000 & 19.0000 & 19.0000 \\
\hline 38 & 18 & 18.0000 & 18.0000 & 18.0000 \\
\hline 41 & 24 & 36.2005 & 1.0543 & 16.6995 \\
\hline
\end{tabular}

Table 2 below summarizes the comparison of estimated development time for Neuro fuzzy model with different neural network models [11]. 
Table 2. Comparison of different models

\begin{tabular}{|c|c|c|c|c|c|}
\hline $\begin{array}{l}\text { Projec } \\
\text { t No. }\end{array}$ & $\begin{array}{l}\text { Actua } \\
1 \mathrm{DT}\end{array}$ & $\begin{array}{l}\text { Estimate } \\
\text { DT using } \\
\text { FFBP NN }\end{array}$ & $\begin{array}{l}\text { Estimate } \\
\text { DT } \\
\text { USING } \\
\text { Cascade } \\
\text { d FFBP } \\
\text { NN }\end{array}$ & $\begin{array}{l}\text { Estimat } \\
\text { ed DT } \\
\text { using } \\
\text { Layer } \\
\text { Recurre } \\
\text { nt NN }\end{array}$ & $\begin{array}{l}\text { Estimate } \\
\text { d DT } \\
\text { using } \\
\text { Trapezo } \\
\text { idal MF } \\
\text { using } \\
\text { Neuro } \\
\text { fuzzy }\end{array}$ \\
\hline 31 & 19 & 9.04 & 9.54 & 9.49 & 19.0000 \\
\hline 32 & 13 & 9 & 9.84 & 9 & 13.0000 \\
\hline 33 & 12 & 9 & 21.91 & 9 & 12.0000 \\
\hline 34 & 12 & 9 & 9 & 9 & 12.0000 \\
\hline 35 & 21 & 21.98 & 22 & 9.21 & 21.0000 \\
\hline 36 & 21 & 21.99 & 22 & 18.89 & 21.0000 \\
\hline 37 & 19 & 21.97 & 21.87 & 9.17 & 19.0000 \\
\hline 38 & 18 & 21.99 & 9.71 & 19.19 & 18.0000 \\
\hline 39 & 24 & 9.31 & 9 & 9.12 & 16.6995 \\
\hline 40 & 25 & 9.31 & 9 & 9.12 & 16.6995 \\
\hline 41 & 18 & 9 & 9 & 9 & 16.6684 \\
\hline
\end{tabular}

Table 3 summarizes the comparison of various neural network models [11] with Neuro fuzzy models using parameters.

Table 3. Comparison of different models using Parameters

\begin{tabular}{|l|l|l|l|}
\hline Models & MMRE (\%) & Pred (25\%) & BRE (\%) \\
\hline FFBP NN & 32.21 & 55 & .6316 \\
\hline $\begin{array}{l}\text { Cascaded } \\
\text { FFBP NN }\end{array}$ & 38.99 & 45 & .7287 \\
\hline $\begin{array}{l}\text { Layer } \\
\text { Recurrent } \\
\text { NN }\end{array}$ & 39.17 & 45 & .8196 \\
\hline $\begin{array}{l}\text { ANFIS } \\
\text { Model using } \\
\text { GMF }\end{array}$ & 25.326 & 72.7273 & .25326 \\
\hline $\begin{array}{l}\text { ANFIS } \\
\text { Model using } \\
\text { Tri MF }\end{array}$ & 22.4146 & 72.7273 & 4.15487 \\
\hline $\begin{array}{l}\text { ANFIS } \\
\text { Model using } \\
\text { Trap MF }\end{array}$ & 6.45624 & 81.8182 & .0921917 \\
\hline
\end{tabular}

A model which gives lower BRE is better than that which gives higher BRE. A model which gives higher Pred (n) is better than that which gives lower Pred (n). A model which gives lower MMRE is better than that which gives higher MMRE. Hence from Table 3 we can observe that ANFIS model using Trapezoidal Membership Function (Trap MF) is better.

Fig 3. Below shows the comparison of different models based on MMRE, Pred (0.25), BRE and we can see that the Pred (0.25) is highest among all the models, so it can be concluded that Neuro Fuzzy model for Trapezoidal MF is the better model for Time estimation among all other models.

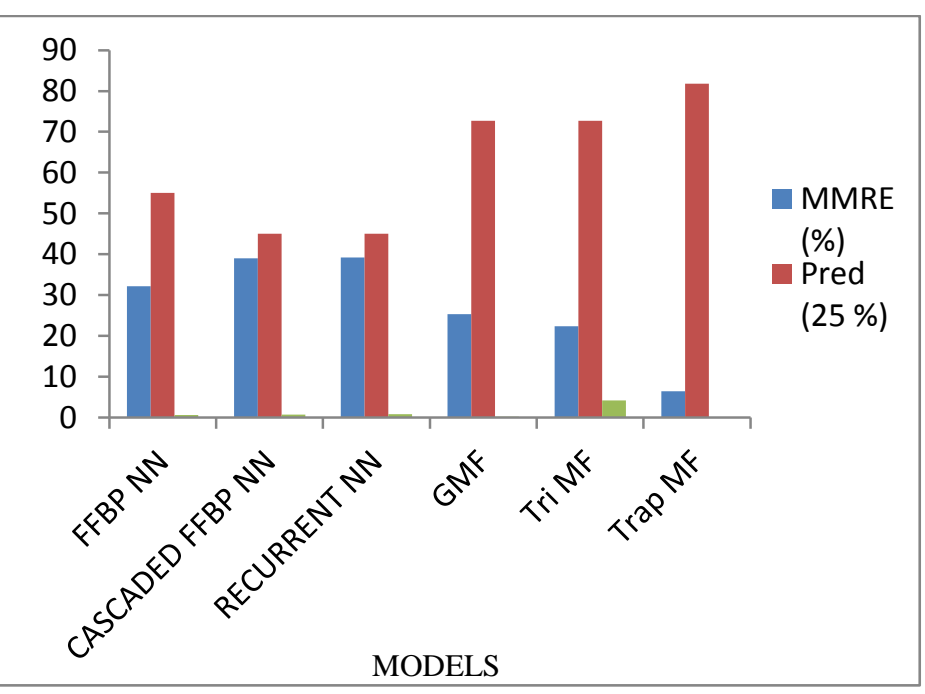

Fig 3: Comparison of different models

\section{CONCLUSION}

The paper suggests a new approach for estimating of software project development time. In this paper, Adaptive Neuro Fuzzy Inference model is considered and three membership functions i.e. Gaussian MF, Triangular MF and Trapezoidal MF are used to predict the future values. It is observed that Neuro Fuzzy model using Trapezoidal membership function gives better results than all other models. It is also observed that Trapezoidal MF gives better results for all the three parameters. In order to achieve more accurate estimation, the estimated values of several other techniques and combine their results may be useful.

\section{ACKNOWLEDGEMENT}

The authors are greatly indebted to the Department of Computer Science and Engineering, D.A.V Institute of Engineering and Technology, Jalandhar for providing excellent lab facilities that make this work possible. 


\section{REFERENCES}

[1] S.Basha and P.Dhavachelvan, "Analysis of Empirical Software Effort Estimation Models" in International Journal of Computer Science and Information Security (IJCSIS), Vol. 7, 2010.

[2] B.Hughes and M.Cotterell, "Software Project Management", Tata McGraw-Hill, 2006.

[3] T.Gruschke, "Empirical Studies of Software Cost Estimation: Training of EffortEstimation Uncertainty Assessment Skills", 11th IEEE International Software Metrics Symposium, IEEE, 2005.

[4] B. W. Boehm, "Software Engineering Economics", Prentice-Hall, Englewood Cliffs, NJ, USA, 1981.

[5] C. C. Kung and J. Y. Su, "Affine Takagi-Sugeno fuzzy modeling algorithm byFuzzy c-regression models clustering with a novel cluster validity criterion", IETControl Theory Appl., pp.1255 - 1265, 2007.

[6] V. Khatibi, Dayang and N. A. Jawawi, "Software Cost Estimation Methods: A Review", Journal of Emerging Trends in Computing and Information Sciences, CIS Journal, Vol. 2, no. 1, ISSN 2079-8407, 2011.

[7] N. Sharma1, A. Bajpai and M. R. Litoriya, "A Comparison of Software Cost Estimation Methods: A Survey", The International Journal of Computer Science and Applications (TIJCSA), Vol.1, no. 3, ISSN - 2278 1080, May 2012.

[8] J. Keung, "Software Development Cost Estimation Using Analogy: A Review", Australian Software Engineering conference, IEEE, 2009.

[9] T. R. Benala, S. Dehuri and R. Mall, "Computational Intelligence in Software Cost Estimation: An Emerging Paradigm", ACM SIGSOFT Software Engineering Notes Page, Vol. 37, no.3, 2012.

[10] J.S. Pahariya, V. Ravi and M. Carr, "Software Cost Estimation using Computational Intelligence Techniques", World Congress on Nature \& Biologically Inspired Computing (NaBIC 2009) IEEE, 2009.

[11] Mrinal Kanti Ghose, Roheet Bhatnagar and Vandana Bhattacharjee, "Comparing Some Neural gNetwork Models for Software Development Effort Prediction", IEEE conference, 2011.

[12] Venus Marza, Amin Seyyedi and Luiz Fernando Capretz, "Estimating Development Time of Software Projects Using a Neuro Fuzzy Approach", World Academy of Science, Engineering and Technology, 2008.

[13] Vachik S. Dave, Kamlesh Dutta, "Neural Network based Software Effort Estimation \& Evaluation criterion MMRE", International Conference on Computer \& Communication Technology (ICCCT), pp.347-351, 2011

[14] Cuauhtémoc López Martín, "Software Development Effort Estimation Using Fuzzy Logic: A Case Study", Proceedings of the Sixth Mexican International Conference on Computer Science (ENC'05), IEEE 2005.

[15] Moataz A. Ahmed, Moshood Omolade Saliu and Jarallah AlGhamdi, "Adaptive fuzzy logic-based framework for software development effort prediction", Information and Software Technology, 2005.
[16] C.J. Burgess, M. Lefley, " Can genetic programming improve software effort estimation? A comparative evaluation", Information and Software Technology, pp.863-873, 2001.

[17] Anish Mittal, Kamal Parkash and Harish Mittal "Software Cost Estimation Using Fuzzy Logic", ACM SIGSOFT Software Engineering Notes Page, Vol 35 no 1, November 2010

[18] Harsh Kumar Verma, Vishal Sharma, "Handling Imprecision in Inputs using Fuzzy Logicto Predict Effort in Software Development", IEEE conference, 2010

[19] G. D. Boetticher, "An assessment of metric contribution in the construction of a neural network-based effort estimator", Proceedings of Second International Workshop on Soft Computing Applied to Software Engineering, 2001

[20] Xishi Huang et al., "A Neuro-Fuzzy Model for Software Cost Estimation", Proceedings of the Third International Conference on Quality Software (QSIC'03), IEEE, 2003.

[21] A.R.Venkatachalam, "Software cost estimation using artificial neural networks", in: Proceedings of the International Joint Conference on Neural Networks, pp. 987-990, 1993

[22] J. Ryder, "Fuzzy modeling of software effort prediction", Proceedings of IEEE Information Technology Conference, Syracuse, NY, 1998.

[23] Allidri et al., "COCOMO Cost Model Using Fuzzy Logic", 7th International Conference On RFuzzy Theory \& Technology Atlantic City, New Jersey, February27March3, 2000.

[24] Heejum Park and Seung Baek, "An Empirical validation of a neural network model for software effor estimation", Expert System with Application,Elsevier, pp.929-937, 2007.

[25] Dinesh C.S. Bisht and Ashok Jangid, "Discharge Modelling using Adaptive Neuro-Fuzzy Inference System", International Journal of Advanced Science and Technology,Vol. 31,pp.99-114, June 2011.

[26] Rama Sree P,Prasad Reddy and Sudha K.R., "Hybrid Neuro-Fuzzy Systems for Software Development Effort Estimation", International Journal on Computer Science and Engineering, Vol. 4, pp.1924-1932, Dec 2012.

[27] Urvashi Rahul Saxena, S.P.Singh, "Software Effort Estimation Using Neuro-Fuzzy Approach", CSI 6th International Conference, pp.1-6, Sept.2012.

[28] Mrinal Kanti Ghose, Roheet Bhatnagar and Vandana Bhattacharjee, "Software Development Effort Estimatiom- Neural Network Vs. Regression Modelling Approach", International Journal of Engineerig and Technology, Vol. 2(7), pp. 2950-2956, 2010.

[29] Divya Kashyap, Ashish Tripathi,Prof. A.K.Mishra, "Software Development Effort and Cost Estimation: Neuro-Fuzzy Model", IOSR Journal of Computer Engineering (IOSRJCE), Vol. 2, pp. 12-14, July-Aug. 2012.

[30] M. Chemuturi, "Software Estimation Best Practices, Tools \& Techniques: A Complete Guide for Software Project Estimator", 2009. 
[31] Vachik S. Dave, Kamlesh Dutta, "Comparison of Regression model, Feed-forward Neural Network and Radial Basis Neural Network for Software Development Effort Estimation", ACM SIGSOFT Software Engineering, Vol. 36, No.5, Sept. 2011.

[32] J. Jantzen, "Neuro- Fuzzy modeling", Reort no 98-H$874,1998$.

[33] M.T. Su, T.C. Ling, "Enhanced Software Development Effort and Cost Estimation Using Fuzzy Logic
Model",Malyasian Journal of Computer Science, Vol.20,No.2,2007,pp.199-207.

[34] A.Heiat, "Comparison of artificial neural network and regression models for estimating software development effort", Information and Software Technology, Vol.44,Issue 15,2002,pp.911-922. 